\title{
Comparison of the contact stress between the sensor and real polyethylene insert in total knee arthroplasty: a finite element analysis
}

\author{
Sang Jun Song, Kang Il Kim, Cheol Hee Park \\ Department of Orthopedic Surgery, College of Medicine, Kyung Hee University, Seoul, Korea \\ Contributions: (I) Conception and design: All authors; (II) Administrative support: SJ Song, KI Kim; (III) Provision of study materials or patients: \\ SJ Song, CH Park; (IV) Collection and assembly of data: CH Park; (V) Data analysis and interpretation: All authors; (VI) Manuscript writing: All \\ authors; (VII) Final approval of manuscript: All authors. \\ Correspondence to: Cheol Hee Park, MD, PhD. Department of Orthopedic Surgery, College of Medicine, Kyung Hee University, 23 Kyunghee-daero, \\ Dongdaemun-gu, Seoul 130-872, Korea. Email: rdohead@hanmail.net.
}

\begin{abstract}
Background: In implants, sensors are made of an acrylic-like plastic, while polyethylene (PE) inserts are made of ultra-high-molecular-weight PE (UHMPE). Thus, the stress distribution on the sensor may be different from that on the $\mathrm{PE}$ insert due to variations in material properties. The present study sought to analyze and compare the stress distribution profile between the sensor and PE insert after total knee arthroplasty (TKA).

Methods: Finite element analysis was performed to estimate contact stress between the sensor and PE insert after TKA. The materials of the femoral component, sensor, and PE insert were determined as cobaltchrome-molybdenum, acryl plastic, and UHMWPE, respectively. The stiffness levels of medial and lateral soft tissue were set at $28.8 \mathrm{~N} / \mathrm{mm}$ and $18.8 \mathrm{~N} / \mathrm{mm}$ at knee flexion and $24.7 \mathrm{~N} / \mathrm{mm}$ and $17.2 \mathrm{~N} / \mathrm{mm}$ at knee extension, respectively. The average and peak contact stress levels on the sensor and $\mathrm{PE}$ were analyzed in knee flexion and extension.

Results: The average amount of contact stress in the medial compartment was $43.4 \mathrm{MPa}$ on the sensor and $3.9 \mathrm{MPa}$ on the $\mathrm{PE}$ insert at knee extension. Meanwhile, the medial compartmental peak contact stress levels were 55.2 $\mathrm{MPa}$ on the sensor and 48.8 MPa on the PE insert at knee extension. The other values of average and peak contact stress among the two materials were less than $5 \mathrm{MPa}$.

Conclusions: There was a difference in the contact stress distribution between the sensor and PE insert due to material properties, especially in the medial compartment at knee extension. The development of a sensor composed of a material with properties similar to a $\mathrm{PE}$ insert would be useful in the prediction of femorotibial contact stress in real implants.
\end{abstract}

Keywords: Knee; arthroplasty; contact stress; polyethylene; sensor

Submitted Mar 31, 2020. Accepted for publication Aug 28, 2020.

doi: $10.21037 /$ atm-20-3011

View this article at: http://dx.doi.org/10.21037/atm-20-3011

\section{Introduction}

A wireless intraoperative load sensor has been created previously to improve the quality of soft tissue balancing during total knee arthroplasty (TKA) (1). It has been designed to reduce polyethylene (PE) wear by facilitating the securement of appropriately balanced loads in the mediolateral compartments (1). Postoperative clinical outcomes are also expected to be improved by proper load balancing using this sensor (1-3).

Stress on an object and its distribution depend on the object's material properties (4). In a TKA implant, the 

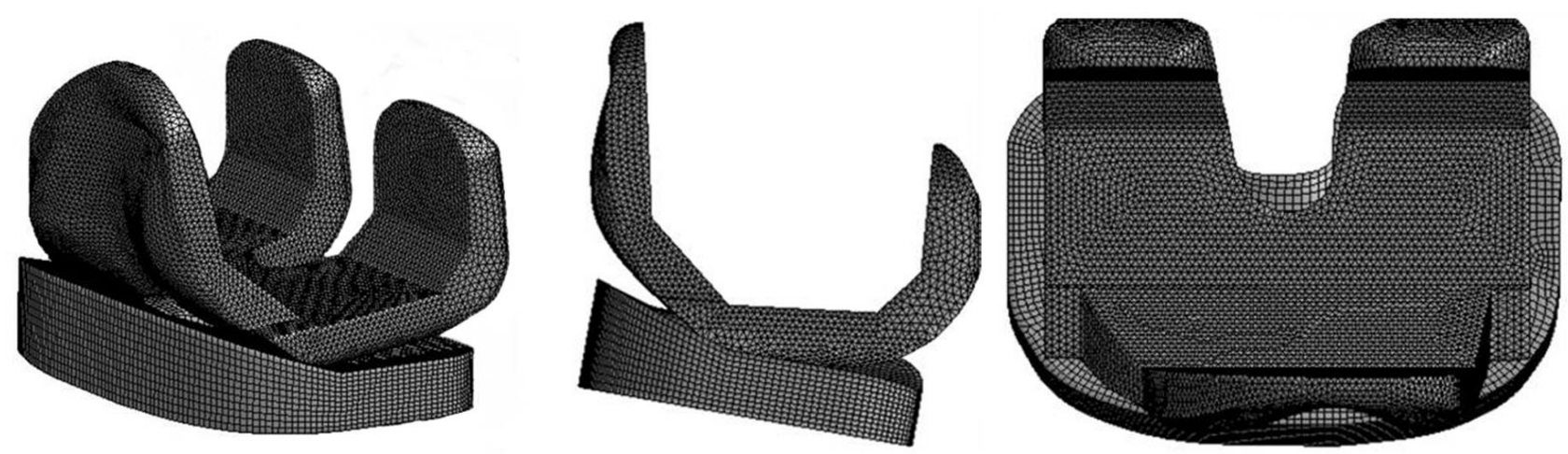

Figure 1 Three-dimensional models of the femoral component, sensor, and PE insert.

sensor is made of an acrylic-like plastic and the PE insert is made of ultra-high-molecular-weight PE (UHMPE). It has been reported that Young's modulus and the Poisson ratio, which are the main indices suggesting the nature of the material's properties, differ between acryl plastic and UHMPE $(5,6)$. Therefore, the distribution of stress on the sensor may be different from that on the PE insert due to variations in material properties. It is unclear as to whether one might be able to infer load balancing on PE inserts of TKA implants from the sensor load data. Currently, there is no method to measure the stress applied to the PE insert in most clinical situations.

Finite element analysis (FEA) is a computer simulation method that enables various analyses by implementing complex geometries and evaluating soft-tissue conditions arithmetically $(7,8)$. It has examined a number of hypotheses that have been difficult to prove by clinical study. Many previous studies in the area of TKA have used FEA to assess the stress placed on the implant within various environments and predict the implant's survival (6,8-11).

If the contact stress and its distribution presented by the sensor do not accurately reflect those of the PE insert, then the involvement of the sensor cannot help to ensure accurate soft-tissue balancing, which is associated with poor clinical outcomes. Accordingly, it would be clinically interesting and important to investigate the stress on the sensor and PE insert in TKA using FEA.

The purpose of the present study was therefore to analyze and compare the stress distribution between a sensor made of acryl plastic and a PE insert made of UHMPE after TKA. It was hypothesized that there would be a difference in the stress distribution between the sensor and $\mathrm{PE}$ insert due to variations in the material properties.

\section{Methods}

A three-dimensional (3D) model of a Nexgen ${ }^{\circledR}$ cruciateretaining TKA implant (Zimmer, Warsaw, IN, USA) was developed with a blueprint provided by the manufacturer (Rapidform version 2006; 3D Systems Korea Inc., Seoul, South Korea) (Figure 1). After creating a 3D model, an FEA program (ABAQUS software version 6.11; Simulia, Province, RI, USA) was used to analyze the contact stress of the 3D FE model. All components including the femoral component, sensor, and PE insert were meshed using fournode tetrahedral elements. The size of the $\mathrm{FE}$ was 0.5 to $1 \mathrm{~mm}$. The numbers of the FE and the node were 200,798 and 120,551 , respectively.

The material for the femoral component was a cobaltchrome-molybdenum alloy (Table 1) (6). The component was modeled as an isotropic elastic homogenous material (11). The size of the femoral component was determined as a D size; this size is commonly chosen for use in our population from among the sizes of the femoral component ranging from $B$ to $G$ provided by the manufacturer (12). The material for the sensor was set as acrylic plastic with an isotropic elastic homogenous material $(5,11)$. The material for the PE insert was UHMWPE (Table 1) and the PE insert was also modeled as an isotropic elastoplastic homogenous material with a yield strength of $30 \mathrm{MPa}$ (10); below the yield point, the PE insert behaved in a purely elastic manner, while, beyond this point, the material exhibited a plastic-like behavior. The sizes of the sensor and tibial component were matched to the dimensions for tibial component size 3. The size of the PE in Nexgen ${ }^{\circledR}$ prostheses is determined by the size of the tibial component, and size 3 is commonly paired with the femoral component size D. The density of the femoral component, sensor, and 
Table 1 Material property in the present FEA

\begin{tabular}{lcc}
\hline & Young's modulus & Poisson ratio \\
\hline $\begin{array}{l}\text { Femoral component (cobalt- } \\
\text { chromium-molybdenum) }\end{array}$ & 195,000 & 0.30 \\
$\begin{array}{l}\text { Sensor (acryl-like plastic) } \\
\begin{array}{l}\text { Polyethylene insert (ultra- } \\
\text { high-molecular-weight } \\
\text { polyethylene) }\end{array}\end{array}$ & 3,200 & 0.35 \\
Soft tissue envelope (ligament) & 1.063 & 0.46 \\
\hline
\end{tabular}

FEA, finite element analysis.

PE insert were determined to $8.28,1.18$, and $0.94 \mathrm{~g} / \mathrm{cm}^{3}$, respectively (13).

It has been established that a femoral component with distal and posterior condyles measuring $9 \mathrm{~mm}$ thick and a sensor or PE insert measuring $9 \mathrm{~mm}$ thick are required to ensure an identical flexion-extension gap. A conventional flexion-extension gap of $17 \mathrm{~mm}$ was established between a rectangular parallelepiped with bony properties (14). The surface of the bony parallelepiped, on which the sensor and PE insert were fixed, was determined to be tilted $7^{\circ}$ posteriorly to attain a posterior tibial slope. The femoral component, sensor, and PE insert were rigidly fixed to the bony material directly.

The soft-tissue ligaments were modeled using an isotropic elastic material (15) and set to be rigidly attached to the bone at the origin and insertion sites. The medial ligament consisted of the anterior and posterior fiber bundles, whereas the lateral ligament was considered to be a single bundle (16). The proximal origins of the medial and lateral ligaments were located at the flexion-extension axis on bony material. The insertion site was located at the bony material site just distal to the center of the medial and lateral border of the sensor or PE insert. The stiffness of the medial and lateral soft ligaments, respectively, was defined differently. We referred to the study by Nagai et al., who reported the difference in stiffness between medial and lateral compartmental soft tissues in Asian patients with degenerative arthritis and varus deformity; the stiffness of the medial and lateral compartmental soft tissues were $24.7 \mathrm{~N} / \mathrm{mm}$ and $17.2 \mathrm{~N} / \mathrm{mm}$ at extension $\left(10^{\circ}\right.$ of knee flexion), and $28.8 \mathrm{~N} / \mathrm{mm}$ and $18.8 \mathrm{~N} / \mathrm{mm}$ at flexion $\left(90^{\circ}\right.$ of knee flexion) (17).

Additionally, a posterior soft tissue was set at the knee extension. This was performed to replicate the posterior condyle pushing the posterior capsule backward when the knee is extended (18). The posteromedial and lateral soft tissues consisted of single bundles, respectively. The origin of the posterior soft tissue was determined as the upper margin level of the posterior femoral condyle at the bone when the femoral component was inserted, while insertion was defined by the low margin of the conventional gap (18). The origin and insertion sites were located at the sagittal midplane of each compartment. The stiffness of the medial and lateral sides of the posterior soft tissue envelope varied but equated to the stiffness of the medial and lateral soft tissue envelopes at extension (posteromedial $v s$. posterolateral $=24.7 v s .17 .2 \mathrm{~N} / \mathrm{mm})(17)$.

The flexion and extension angles were determined with vertical axes between the femoral component and the sensor or PE insert. At knee extension, the tangent surface of the most posterior edges of the femoral and tibial components was aligned in the vertical dimension. Meanwhile, the centerline of the femoral component was aligned to that of the tibial component at knee flexion and extension. Contact was detected when the perpendicular distance between the femoral component and PE insert was less than $0.01 \mathrm{~mm}$ (19). The contact area between the femoral component and $\mathrm{PE}$ insert was determined to be maximal, ensuring matching between the components.

The average contact stresses on the sensor and PE insert were analyzed at knee flexion and extension, which were representative values for the stress in each compartment. Additionally, the peak contact stresses on both materials were also analyzed at knee flexion and extension. Any external force, beyond the ligament forces, was not determined to exist because the situation during surgery was reproduced (14).

\section{Results}

Overall, the distribution of the average and peak contact stresses on the sensor in our FEA study were consistent with the load distribution evaluated with the sensor after conventional gap-balancing in CR TKA using the Nexgen ${ }^{\circledR}$ prosthesis as reported before in a previous study (14). The medial compartmental load was evidently high at the knee extension in the previous study.

The average contact stress in the medial compartment was $43.4 \mathrm{MPa}$ on the sensor and $31.9 \mathrm{MPa}$ on the PE insert at knee extension (Table 2, Figure 2). The peak contact stress in the medial compartment was $55.2 \mathrm{MPa}$ on the sensor and $48.8 \mathrm{MPa}$ on the $\mathrm{PE}$ insert at knee extension (Table 2, Figure 2). The average and peak stresses in the 
Table 2 The average and peak contact stresses

\begin{tabular}{|c|c|c|c|c|c|c|}
\hline Stress & \multicolumn{3}{|c|}{ Sensor (acryl plastic) } & \multicolumn{3}{|c|}{ Polyethylene (UHMPE) } \\
\hline \multicolumn{7}{|c|}{ Average stress (MPa) } \\
\hline Extension $^{\dagger}$ & 43.4 & 26.5 & 16.9 & 31.9. & 22.4 & 9.5 \\
\hline Flexion $^{\ddagger}$ & 31.5 & 17.8 & 13.7 & 31.0 & 15.0 & 16.0 \\
\hline Extension $^{\dagger}$ & 55.2 & 33.2 & 22.0 & 48.8 & 36.9 & 11.9 \\
\hline Flexion $^{\ddagger}$ & 44.4 & 25.9 & 18.5 & 41.5 & 22.3 & 19.2 \\
\hline
\end{tabular}

${ }^{\dagger}, 10^{\circ}$ of knee flexion; ${ }^{\ddagger}, 90^{\circ}$ of knee flexion.

A Extension (10 degree of flexion)

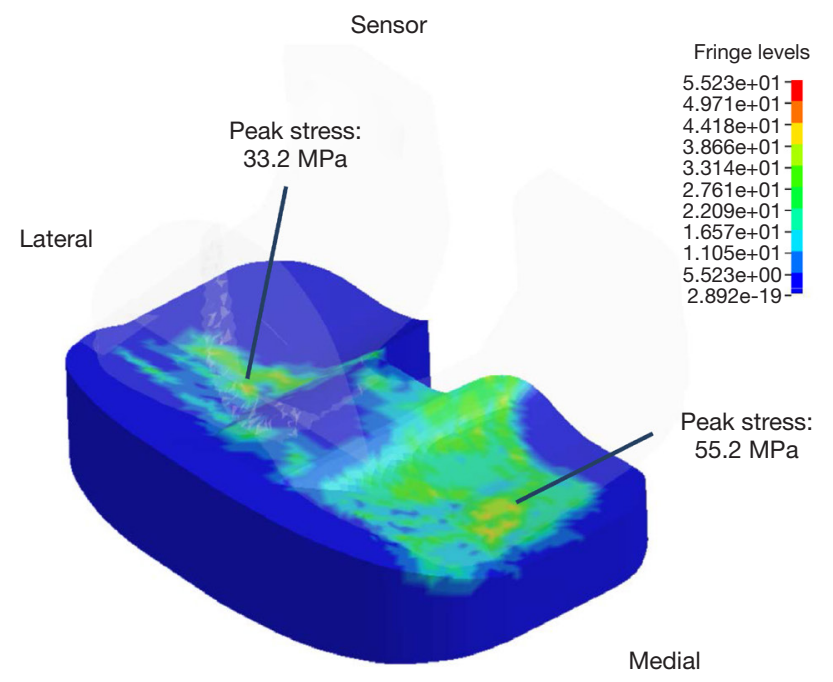

B Flexion (90 degree of flexion)

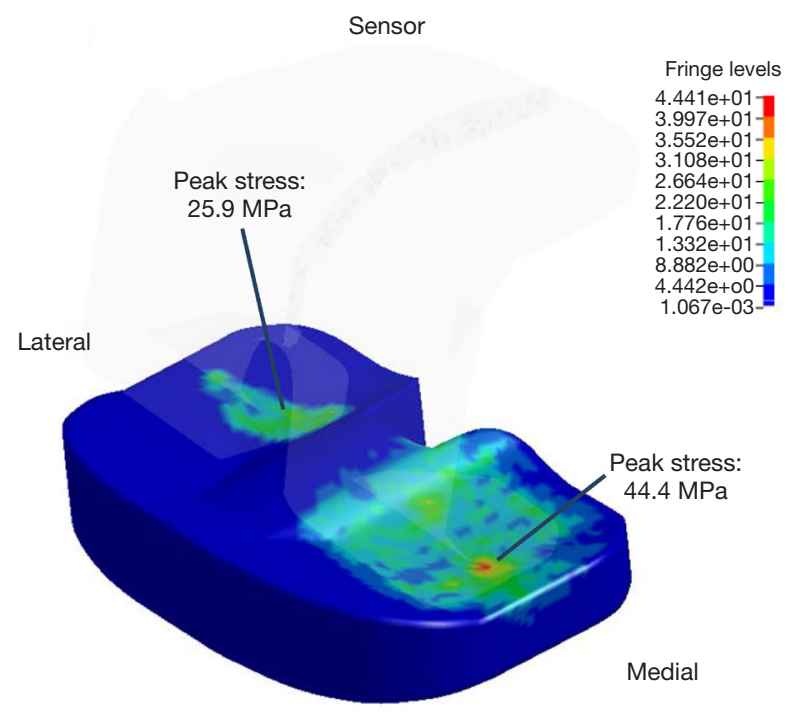

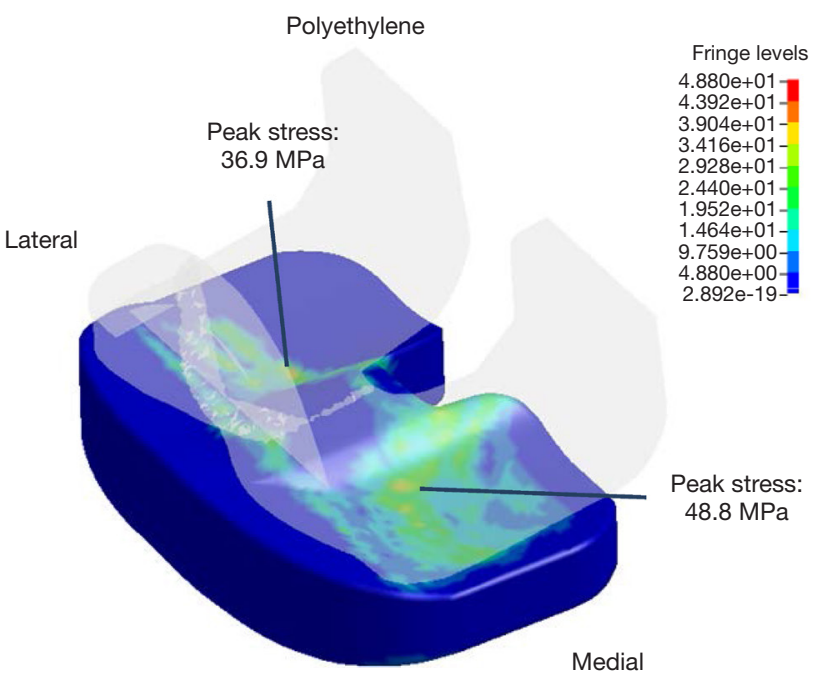

Polyethylene

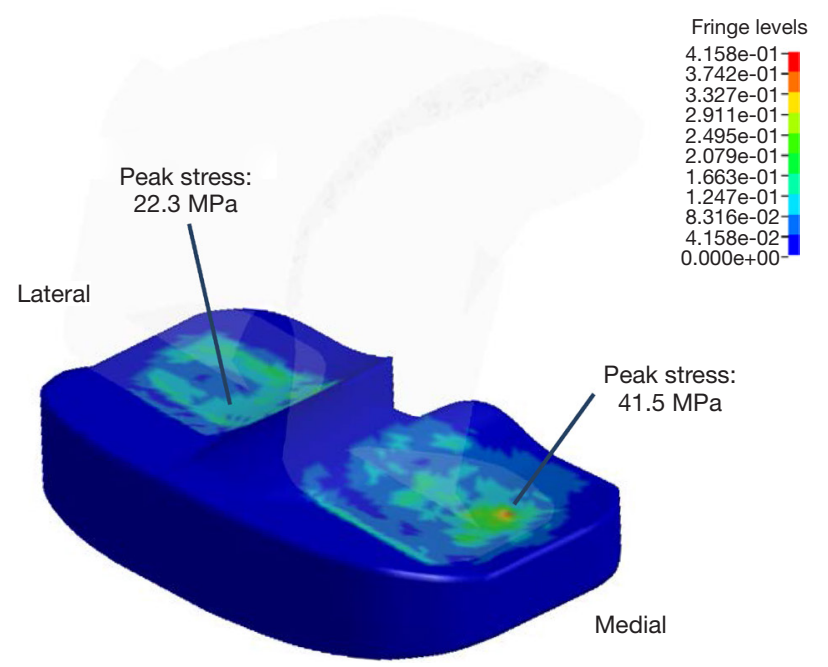

Figure 2 The contact stress on the sensor and PE insert at knee extension (A) and flexion (B). PE, polyethylene. 
medial compartment on the sensor were 5-10 MPa greater than those on the PE insert at knee extension, which could be significant difference.

The other values of average and peak contact stress between the two materials were less than $5 \mathrm{MPa}$, including those values recorded from the lateral compartment at knee flexion and extension and the medial compartment at knee flexion.

\section{Discussion}

The most important finding of the present study was that there were differences in the average and peak contact stresses between the sensor and PE insert after achieving a rectangular gap in TKA. These differences were evident in the medial compartment at knee extension.

Song et al. (14) demonstrated that mediolateral load imbalances with greater medial compartmental load existed as determined by the sensor after conventional gapbalancing with a tensiometer. Such a mediolateral load imbalance was especially pronounced at knee extension. The cause of this load imbalance was explained by the difference between the degrees of the stiffness of the medial and lateral soft tissues in the arthritic knee (17). The stress on the sensor in our FEA study with different medial and lateral soft tissue stiffness values was consistent with the load distribution observed in a previous study (14). The average stress was higher in the medial compartment than in the lateral compartment after conventional gap-balancing. The mediolateral difference between the average stresses was greater at knee extension than at knee flexion. However, the medial compartmental stress on the PE insert was not as large as that on the sensor at knee extension in our FEA study. Separately, the difference in average stress between the medial and lateral compartments at knee extension on $\mathrm{PE}$ was not as large as that on the sensor.

As described, the sensor is made of acrylic plastic and the PE insert is made of UHMWPE. Considering the properties of the two materials, the $\mathrm{PE}$ insert exhibits a more pronounced elasticity relative to the sensor $(5,6)$. In addition, the PE insert boasts the capability to deform its shape under high stress (10). Therefore, when the PE insert is subjected to high stress, it could be deformed to be more congruent with the femoral component compared to the sensor being made of acrylic plastic, which may counteract substantial increases in contact stress $(19,20)$.

In the present study, the average and peak contact stress in the medial compartment of the sensor were 5 to $10 \mathrm{MPa}$ greater than the contact stress of the PE inserts at knee extension. It was reported that the risk of damage for the $\mathrm{PE}$ insert increases with increasing contact stress of 5 to $10 \mathrm{MPa}$ (21). Accordingly, the range of 5 to $10 \mathrm{MPa}$ could be a valuable criterion for assessing differences in the contact stresses between the sensor and PE insert. It is thought that the difference in contact stress of the medial compartment at extension between the sensor and PE will be noteworthy.

An intraoperative load sensor is recognized as a useful device to allow objective soft tissue-balancing in TKA. Recent studies have reported that sensor-assisted TKAs with load balancing show more favorable clinical outcomes postoperatively (1-3). However, sensor users should be aware that contact stress can be different between the sensor and PE insert. The load indicated by the sensor could be remarkably greater than that placed on the $\mathrm{PE}$ insert in the medial compartment at knee extension, despite a rectangular gap existing after TKA. Accordingly, surgeons using the sensor should be careful not to over-release the medial soft tissue, especially at extension. Additionally, it may be necessary to develop a sensor composed of a material with properties similar to those of the PE insert.

The present study has several limitations. First, this FEA study did not accurately reproduce all the specific anatomical features of the knee including the capsule, ligament, tendon, and muscle. However, it is thought that the results of this study validate our implementation given the contact stress distribution of the sensor in this study was similar to the load distribution of the sensor reported in a previous clinical study (14). More accurate data can be obtained if the soft-tissue properties and anatomy of an arthritic knee are accurately reproduced. Second, not all of the implants available for TKA were reproduced in our FEA; for example, implementation of the tibial component was omitted. However, despite this problem, achieving the main aim of comparing the contact stresses of two materials under the same stress condition was not significantly impaired. Third, the present study did not investigate contact stress in dynamic conditions characterized by muscle action and weight-bearing. Recently, FEA has shown validity in developing a dynamic model of insertfemoral contact able to efficiently determine joint and contact mechanics simultaneously during dynamic loading conditions (22). However, the main purpose of this study was to investigate whether the intraoperative contact stress on the sensor is comparable to that on the PE insert. Fourth, one FEA model was used for contact stress analysis. 
An analysis performed using a number of FEA models may produce more robust data. Fifth, this study did not perfectly implement the sensor device. Although the sensor is a device composed of an acrylic plastic surrounding the electronic instrument, we reproduced the sensor with a homogenous material made of acryl plastic. However, the property of the contact surface is the most significant factor in contact stress and it was reproduced appropriately in this study. Lastly, the present FEA study reproduced one specific type of TKA prosthesis. It is known that implant design influences the contact stress (23). Shu et al. (24) showed that the contact mechanics depended on the sagittal conformity between the femoral component and PE insert. Ardestani et al. (25) reported that the femoral and tibial distal or posterior radii and frontal radius impacted the contact pressure and its distribution on the PE insert. Further analysis using other prostheses will be necessary.

\section{Conclusions}

There was a difference in the contact stress distribution between the sensor and $\mathrm{PE}$ insert due to material properties, especially in the medial compartment at knee extension. The development of a sensor composed of a material with properties similar to a $\mathrm{PE}$ insert would be useful in the prediction of femorotibial contact stress in real implants.

\section{Acknowledgments}

Funding: None.

\section{Footnote}

Conflicts of Interest: All authors have completed the ICMJE uniform disclosure form (available at http://dx.doi. org/10.21037/atm-20-3011). The authors have no conflicts of interest to declare.

Ethical Statement: The authors are accountable for all aspects of the work in ensuring that questions related to the accuracy or integrity of any part of the work are appropriately investigated and resolved. Ethical approval is not required as this is a FEA study.

Open Access Statement: This is an Open Access article distributed in accordance with the Creative Commons Attribution-NonCommercial-NoDerivs 4.0 International License (CC BY-NC-ND 4.0), which permits the non- commercial replication and distribution of the article with the strict proviso that no changes or edits are made and the original work is properly cited (including links to both the formal publication through the relevant DOI and the license). See: https://creativecommons.org/licenses/by-nc-nd/4.0/.

\section{References}

1. Gustke KA, Golladay GJ, Roche MW, et al. A new method for defining balance: promising short-term clinical outcomes of sensor-guided TKA. J Arthroplasty 2014;29:955-60.

2. Geller JA, Lakra A, Murtaugh T. The Use of Electronic Sensor Device to Augment Ligament Balancing Leads to a Lower Rate of Arthrofibrosis After Total Knee Arthroplasty. J Arthroplasty 2017;32:1502-4.

3. Chow JC, Breslauer L. The Use of Intraoperative Sensors Significantly Increases the Patient-Reported Rate of Improvement in Primary Total Knee Arthroplasty. Orthopedics 2017;40:e648-51.

4. Brown TD, Ferguson AB Jr. Mechanical property distributions in the cancellous bone of the human proximal femur. Acta Orthop Scand 1980;51:429-37.

5. EngineeringToolBox. Young's Modulus - Tensile and Yield Strength for common Materials. 2003. Available online: https://www.engineeringtoolbox.com/youngmodulus-d_417.html

6. Kang KT, Son J, Kwon OR, et al. Malpositioning of Prosthesis: Patient-specific Total Knee Arthroplasty Versus Standard Off-the-Shelf Total Knee Arthroplasty. J Am Acad Orthop Surg Glob Res Rev 2017;1:e020.

7. Pfeiffer FM. The Use of Finite Element Analysis to Enhance Research and Clinical Practice in Orthopedics. J Knee Surg 2016;29:149-58.

8. Popescu R, Haritinian EG, Cristea S. Relevance of Finite Element in Total Knee Arthroplasty - Literature Review. Chirurgia (Bucur) 2019;114:437-42.

9. Avanzini AJM, Design. Mechanical characterization and finite element modelling of cyclic stress-strain behaviour of ultra high molecular weight polyethylene. Materials Design 2008;29:330-43.

10. Kang KT, Kwon SK, Kwon OR, et al. Comparison of the biomechanical effect of posterior condylar offset and kinematics between posterior cruciate-retaining and posterior-stabilized total knee arthroplasty. Knee 2019;26:250-7.

11. Mou Z, Dong W, Zhang Z, et al. Optimization of parameters for femoral component implantation during 
TKA using finite element analysis and orthogonal array testing. J Orthop Surg Res 2018;13:179.

12. ZimmerBiomet. Nexgen Cruciate Retaining (CR) and Revision Instrumentation Surgical Technique 2017. Available online: https://www.zimmerbiomet.com/ content/dam/zimmer-biomet/medical-professionals/000surgical-techniques/knee/cruciate-retaining-cr-revisioninstrumentation-surgical-technique-for-cruciateretaining-augmentable-cpa-knees-surgical-technique.pdf

13. Kitisak K, Siroros N, Rungsiyakull CJIJoPM, et al. Finite Element Analysis in Posterior Stabilized Total Knee Arthroplasty on Kneeling. Available online: http://www. ijpmbs.com/papers/11-E0008.pdf

14. Song SJ, Lee HW, Kim KI, et al. Load imbalances existed as determined by a sensor after conventional gap balancing with a tensiometer in total knee arthroplasty. Knee Surg Sports Traumatol Arthrosc 2020;28:2953-61.

15. Ren D, Liu Y, Zhang X, et al. The evaluation of the role of medial collateral ligament maintaining knee stability by a finite element analysis. J Orthop Surg Res 2017;12:64.

16. Tanaka Y, Nakamura S, Kuriyama S, et al. Medial tilting of the joint line in posterior stabilized total knee arthroplasty increases contact force and stress. Clin Biomech (Bristol, Avon) 2018;53:54-9.

17. Nagai K, Muratsu H, Matsumoto T, et al. Soft tissue balance changes depending on joint distraction force in total knee arthroplasty. J Arthroplasty 2014;29:520-4.

18. Hananouchi T, Yamamoto K, Ando W, et al. The

Cite this article as: Song SJ, Kim KI, Park CH. Comparison of the contact stress between the sensor and real polyethylene insert in total knee arthroplasty: a finite element analysis. Ann Transl Med 2020;8(21):1424. doi: 10.21037/atm-20-3011 intraoperative gap difference (flexion gap minus extension gap) is altered by insertion of the trial femoral component. Knee 2012;19:601-5.

19. D'Lima DD, Steklov N, Fregly BJ, et al. In vivo contact stresses during activities of daily living after knee arthroplasty. J Orthop Res 2008;26:1549-55.

20. Szivek JA, Anderson PL, Benjamin JB. Average and peak contact stress distribution evaluation of total knee arthroplasties. J Arthroplasty 1996;11:952-63.

21. Scuderi G, Tria A. Surgical techniques in total knee arthroplasty. 1st ed. Springer Science \& Business Media, 2006.

22. Halloran JP, Petrella AJ, Rullkoetter PJ. Explicit finite element modeling of total knee replacement mechanics. J Biomech 2005;38:323-31.

23. Medel FJ, Martínez-Morlanes MJ, Alonso PJ, et al. Microstructure, thermooxidation and mechanical behavior of a novel highly linear, vitamin E stabilized, UHMWPE. Mater Sci Eng C Mater Biol Appl 2013;33:182-8.

24. Shu L, Yamamoto K, Kai S, et al. Symmetrical cruciateretaining versus medial pivot prostheses: The effect of intercondylar sagittal conformity on knee kinematics and contact mechanics. Comput Biol Med 2019;108:101-10.

25. Ardestani MM, Moazen M, Jin Z. Contribution of geometric design parameters to knee implant performance: Conflicting impact of conformity on kinematics and contact mechanics. Knee 2015;22:217-24. 